Ingeniería Investigación y Tecnología, ISSN 2594-0732 V. 4. 279-290, 2004

(artículo arbitrado)

DOI:http://dx.doi.org/10.22201/fi.25940732e.2004.05n4.018

\title{
Impedancia propia de dipolos cortos con geometrías cónicas
}

\author{
A. Monsiváis-Huertero y R. Neri-Vela \\ Departamento de Telecomunicaciones, \\ Facultad de Ingeniería, U N A M \\ E-mails: alejandromonsivais@ hotmail.com y aldeca@marconi.fi-b.unam.mx
}

(recibido: julio de 2002; aceptado: septiembre de 2003)

\section{Resumen}

En este artículo se proporcionan datos sobre la impedancia propia de dipolos cortos y curvos que permiten verificar la exactitud de un programa de computadora personal hecho para analizar las características de radiación de una antena determinada por el método de momentos. En particular, estos datos sirven para comprobar los elementos complejos de la diag onal prin cipal de la matriz de impedancias obtenida por dicho método para una gran diversidad de antenas con geometría cónica. Adicionalmente, los resultados obtenidos demuestran que el error introducido al aproximar cada dipolo curvo por uno recto es despreciable, siempre y cuando su radio de curvatura sea igual o mayor que 2 .

Descriptores: dipolo corto, dipolo curvo, impedancia propia, método de momentos, geometría cónica.

\begin{abstract}
$D$ ata on theself im ped ance of short and bent di poles arepro vided in this pa per, that areusful to ver ify the effectiveness of com puter programswritten to an alyzethera diation character is tics of a deter mined an tenna by the M o ment $M$ ethod. Par ticu larly, these data areuseful to check theel ements of themain di ag o nal of the com plex im ped ancema trix that is ob tained by this method, and can beused for many an ten nas of differ ent con i cal geom etry. Ad di tionally, theresults ob tained show that theer ror in tro duced when ap prox i mat ing each bent di poleb y a straight one is neg li gi ble, as long as its cur va ture ra dius is equal or greater than 2.
\end{abstract}

Keywords: short di pole, bent di pole, self-impedance, mo ment method, con i cal geom etry.

\section{Introducción}

En todo sistema de comunicación inalámbrica es necesario caracterizar las antenas por medio de sus propiedades de radiación. Existen varios métodos numéricos que facilitan estos cálculos, uno de ellos es el Método de Mo- mentos, que divide a la antena bajo estudio en $\mathrm{N}$ segmentos, considerados como dipo- los cortos, mismos que pueden o no tener la misma longitud. Cada segmento tiene una impedancia propia asociada, y debido a la 
proximidad física entre todos los segmentos del con duc tor, también existe una impedancia mutua entre cada par de ellos (Harrington, 1968).

En otro artículo publicado en esta misma revista (Neri, 1999) se explica todo el procedimiento matemático para obtener la matriz de impedancias de cualquier antena lineal arbitraria por el Método de Momentos (MM); asimismo, se incluyen algunos resultados para antenas tipo Yagi-Uda, con todos sus elementos rectos. Sin embargo, no todas las antenas lineales consisten de elementos rectos, sino que pueden tener diversas curvaturas. Ejemplos típicos (Figura 1) son el dipolo doblado (Hernández et al., 2001) , la antena circular y la antena parabólica de rejilla o tubular (Neri y Maclean, 1978).

Uno de los mayores problemas que enfrenta un investigador 0 un alumno de la asignatura antenas y propagación, es cuando desea emplear el MM por primera vez, e ir adquiriendo experiencia y certidumbre en sus resultados, es cómo verificar si la matriz de impedancias calculada es correcta o no antes de invertirla y proseguir con la obtención de las corrientes y los campos radiados. Para el caso de los conductores rectos sí existe una referencia que permite comprobar dichas impedancias y la eficacia del programa de computadora que haya

sido elaborado en forma personal (Neri, 1980). Sin embargo, para el caso de elementos curvos no hay datos disponibles en la literatura y queda la duda sobre qué tanto influye la curvatura o si la impedancia propia es igual a la de un dipolo recto por el hecho de que ambos sean muy cortos. Por tanto, en este trabajo se aporta información que permita verificar, como primer paso, la di ago nal prin ci pal de la matriz compleja tomando en cuenta la curvatura de la antena. En esta diagonal principal están todas las impedancias propias de los segmentos en que la antena ha sido subdividida; si todos éstos son de la misma longitud y curvatura, por ejemplo en una antena de lazo cir cu lar, entonces basta con conocer una sola impedancia propia, ya que todas las demás de la diagonal principal deben ser iguales. Pero si los segmentos cambian de curvatura en diferentes secciones de la antena, como por ejemplo en cada conductor parabólico de los que forman a la antena mostrada en la Figura 1c, entonces las impedancias de la di ag o nal prin ci pal ya no son necesariamente idénticas. Los resultados aquí presentados permiten verificarlas una a una, conforme su radio de curvatura varía, al igual que las impedancias propias de los segmentos de otras antenas curvas.

\section{Desarrollo}

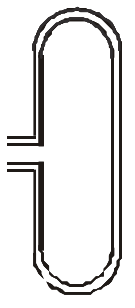

( a )

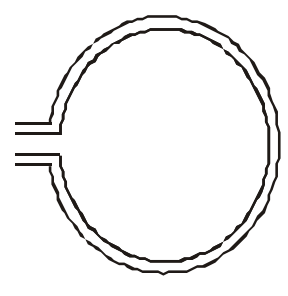

(b)

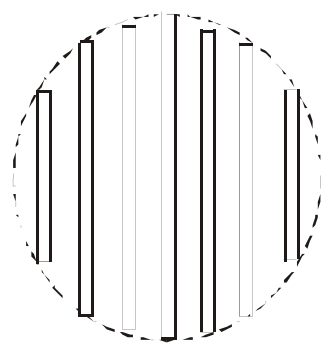

(c)

Figura 1. Ejemplos de antenas lineales formadas con elementos tubulares curvos: (a) dipolo doblado, (b) lazo circular, (c) paraboloide de rejilla visto de frente 
En este análisis general de la impedancia propia de dipolos cortos y curvos, es decir, con geometría cónica, conviene trabajar en coordenadas polares. Una cónica se define como la curva trazada por un punto $\mathrm{P}$ que se mueve de tal manera que la distancia entre este punto móvil y otro punto fijo (llamado foco), dividida entre la distancia de $\mathrm{P}$ a una recta fija (llamada directriz) resulta ser una razón constante. La razón constante es la excentricidad e de la curva (Solís et al., 1995). En la figura 2 se muestra esta geometría, en donde el foco coincide con el origen y el eje focal es colineal con el eje polar. La ecuación que representa a esta cónica en coordenadas polares es (Spiegel et al., 2000):

$$
r=\frac{p}{1-e \cos \theta}
$$

Donde $p$ es la distancia del foco a la directriz y $r$ es la distancia del foco a un punto de la cónica e igual a la magnitud de $\mathbf{R}(\theta)$.

Ahora bien, la función vectorial en coordenadas cartesianas que representa a la misma cónica está dada por la expresión:

$$
\mathbf{R}=x \mathbf{a}_{x}+y \mathbf{a}_{y}
$$

0 bien, en términos de la variable $\theta$ y sustituyendo la ecuación (1) en la ecuación (2), con $p$ y r en lon gi tudes de onda:

$$
\mathbf{R}(\theta)=\frac{p_{\lambda} \cos \theta}{1-e \cos \theta} \mathbf{a}_{x}+\frac{p_{\lambda} \operatorname{sen} \theta}{1-e \cos \theta} \mathbf{a}_{y}
$$

donde $\mathbf{R}$ es el vec tor de posición de la función vec to rial y $\mathbf{a}_{x}$ y $\mathbf{a}_{y}$ son los vectores unitarios del sistema (Figura 2).

Como se muestra en la figura 3, para poder aplicar el MM, es necesario caracterizar a cada conductor tubular de la antena por medio de una curva que represente a la curvatura del segmento sobre su eje y otra que represente a la curvatura sobre su superficie.

La distancia $R_{m}$ se mide desde un punto fuente sobre el eje de cada segmento $n$ hacia

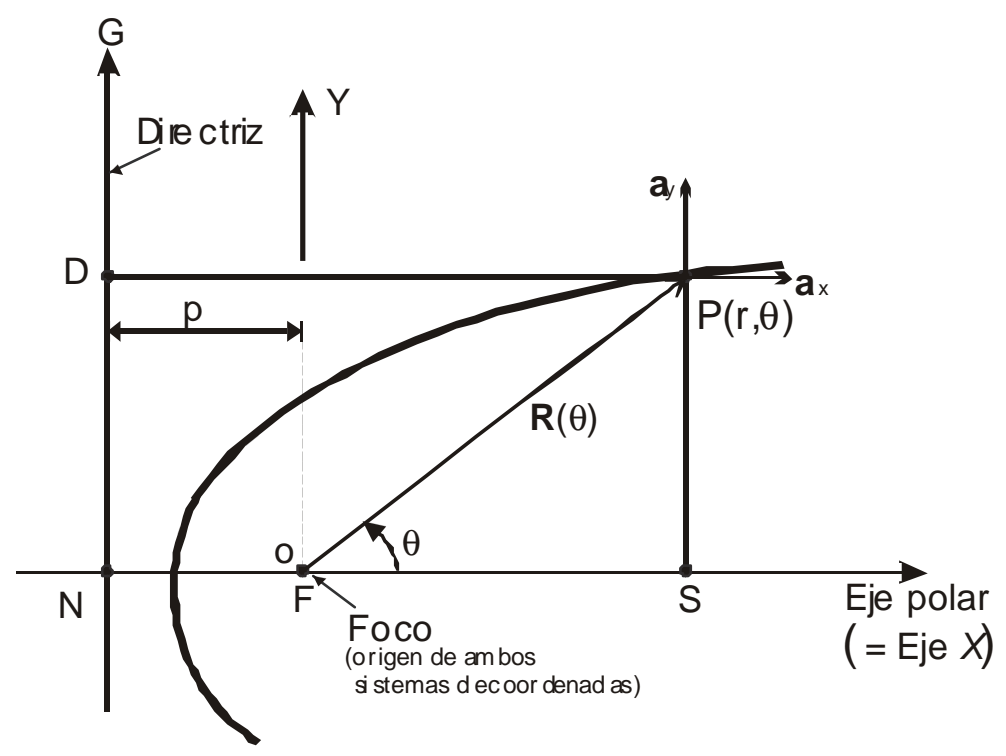

Figura 2. Geometría de una cónica en los sistemas polar y cartesiano 


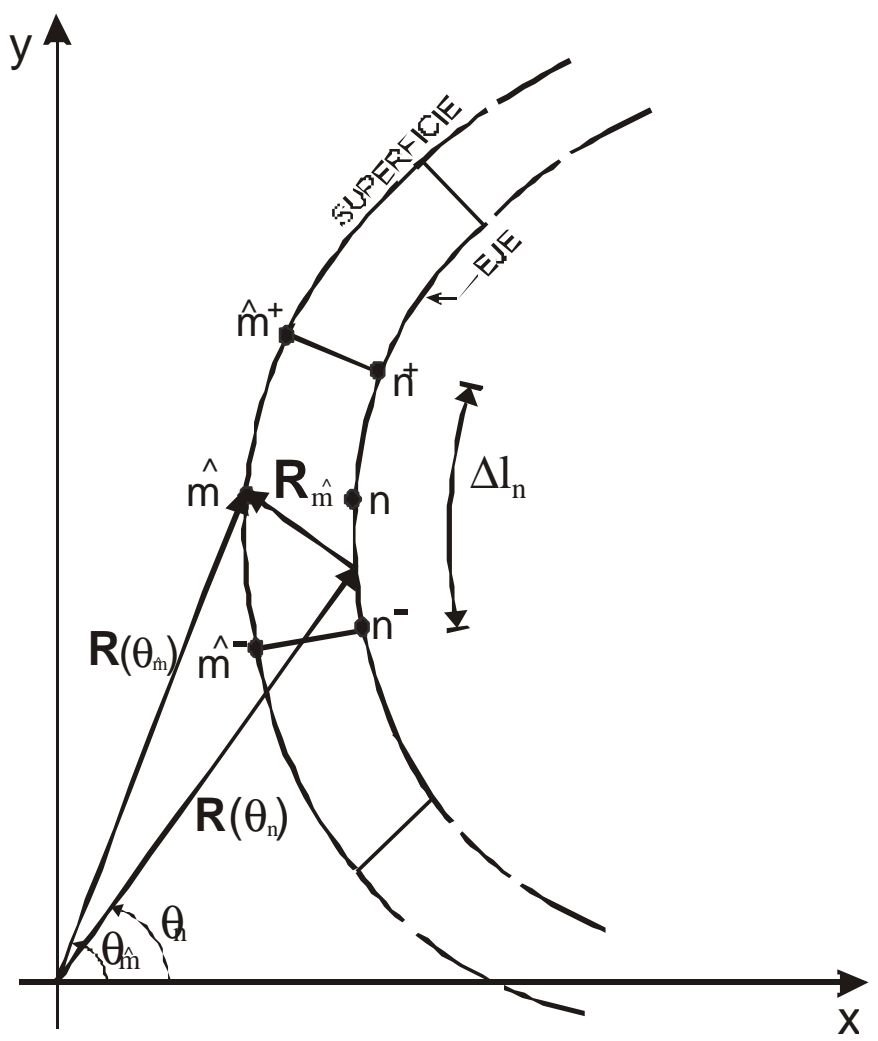

Figura 3. Modelo de un segmento curvo a lo largo del conductor. Sólo se indican el eje y una de las dos superficies laterales

el punto central sobre la superficie del segmento $\hat{m}$, y se calcula como la magnitud de la siguiente ecuación vectorial:

(4)

$$
R_{m}=\left|\mathbf{R}\left(\theta_{m}\right)-\mathbf{R}\left(\theta_{n}\right)\right|
$$

donde $\theta_{\mathrm{m}}$ y $\theta_{\mathrm{n}}$ son los ángulos que determinan la posición del punto cen tral del segmento $\hat{\mathrm{m}} \mathrm{y}$ el punto fuente sobre el eje del segmento $n$, respectivamente.

Si la magnitud de $\mathbf{R}\left(\theta_{\mathrm{m}}\right)$ y $\mathbf{R}\left(\theta_{\mathrm{n}}\right)$ se representa, respectivamente, como $r_{m}$ y $r_{n}$, la ecuación 4 en coordenadas polares es equivalente a la distancia entre dos puntos:

$$
R_{m}=\sqrt{r_{m}^{2}+r_{n}^{2}-2 r_{m} r_{n} \cos \left(\theta_{m}-\theta_{n}\right)}
$$

Donde:

$$
r_{\hat{m}}=\frac{p_{\lambda}}{1-e \cos \theta_{\mathrm{m}}}
$$

$$
r_{n}=\frac{p_{\lambda}}{1-e \cos \theta_{n}}
$$


y $R_{m}, r_{m}$ y $r_{n}$ están en lon gi tudes de onda.

Para aplicar las ecuaciones que permiten calcular las impedancias propias (Neri, 1999) es necesario conocer el ángulo $\beta$ formado entre el vec tor tangente a la superficie del conductor en el segmento $\hat{m}$ y el vector de potencial magnético $\mathbf{A}$ en el mismo segmento; dicho vector $\mathbf{A}$ es producido por la corriente que fluye en el segmento $n$ y tiene la misma dirección que la corriente en este último segmento. Por lo tanto, este ángulo $\beta$ puede ser calculado por medio de la definición del producto escalar (Antón, 1998):

$$
\mathbf{R}^{\prime}\left(\theta_{\mathrm{m}}\right) \cdot \mathbf{R}^{\prime}\left(\theta_{\mathrm{n}}\right)=\mathbf{R}^{\prime}\left(\theta_{\mathrm{m}}\right)\left|\mathbf{R}^{\prime}\left(\theta_{\mathrm{n}}\right)\right| \cos \beta
$$

de donde

$$
\cos \beta=\frac{\mathbf{R}^{\prime}\left(\theta_{\mathrm{m}}\right) \cdot \mathbf{R}^{\prime}\left(\theta_{\mathrm{n}}\right)}{\left|\mathbf{R}^{\prime}\left(\theta_{\mathrm{m}}\right)\right| \mathbf{R}^{\prime}\left(\theta_{\mathrm{n}}\right) \mid}
$$

En la cual $\mathbf{R}^{\prime}\left(\theta_{\mathrm{m}}\right)$ y $\mathbf{R}^{\prime}\left(\theta_{\mathrm{n}}\right)$ son los vectores tangentes a los segmentos $\hat{m}$ y $n$, respectivamente (O'Neil, 1999). Para obtener estos vectores tangentes es necesario derivar la ecuación (3), por lo que se tiene:

$$
\begin{gathered}
\mathbf{R}^{\prime}(\theta)=\left[\frac{-p_{\lambda} \operatorname{sen} \theta}{(1-e \cos \theta)^{2}}\right] \mathbf{a}_{x}+ \\
{\left[\frac{\left.p_{\lambda} \cos \theta-e p_{\lambda}\right]}{(1-e \cos \theta)^{2}}\right] a_{y}} \\
\mathbf{R}^{\prime}(\theta)=\frac{p_{\lambda} \sqrt{1-2 e \cos \theta+e^{2}}}{(1-e \cos \theta)^{2}}
\end{gathered}
$$

1 Esta expresión se maneja en el análisis matemático como una ecuación diferencial, ya que si se quiere encontrar su ecuación primitiva es necesario un método numérico para resolver la integral elíptica que se presenta.
Sustituyendo las ecuaciones (8) y (9) en (7) se puede conocer el valor de $\beta$. Después de efectuar las derivadas necesarias y simplificar, el coseno de dicho ángulo es:

$$
\cos \beta=\frac{A+B}{C D}
$$

Donde:

$$
A=p_{\lambda} \operatorname{sen} \theta_{n}\left[p_{\lambda} \operatorname{sen} \theta_{m}+\frac{d}{2} \operatorname{sen} \theta_{m}\left(1-e \cos \theta_{m}\right)^{2}\right]
$$

$$
B=\left(p_{\lambda} \cos \theta_{n}-e p_{\lambda}\right)
$$

$$
\left[p_{\lambda} \cos \theta_{m}-e p_{\lambda}+\frac{d}{2} \cos \theta_{m}\left(1-e \cos \theta_{m}\right)^{2}\right]
$$

$$
C=\sqrt{p_{\lambda}^{2}-2 p_{\lambda}^{2} e \cos \theta_{n}+e^{2} p_{\lambda}^{2}}
$$

$$
\begin{aligned}
& D=\sqrt{\left[p_{\lambda} \operatorname{sen} \theta_{m}+\frac{d}{2} \operatorname{sen} \theta_{m}\left(1-e \cos \theta_{m}\right)^{2}\right]^{2}}+ \\
& \sqrt{\left[p_{\lambda} \operatorname{sen} \theta_{m}-e p_{\lambda}+\frac{d}{2} \operatorname{sen} \theta_{m}\left(1-e \cos \theta_{m}\right)^{2}\right]^{2}}
\end{aligned}
$$

(10d)

en donde $d$ es el diámetro del tubo de la antena en lon gi tudes de onda.

La expresión de la diferencial dl a lo largo del eje del segmento $n$ también es necesaria para efectuar las integrales de longitud requeridas en el Método de Momentos, y puede ser obtenida con la longitud de arco de una curva, definida diferencialmente en 
coordenadas polares por la expresión (0'Neil, 1999):

$$
(d l)^{2}=(d r)^{2}+(r d \theta)^{2}
$$

Al sustituir la ecuación (1) en la (11), se tiene que1:

$$
d l=\frac{p \sqrt{1-e^{2} \operatorname{sen}^{2} \theta}}{1-e \operatorname{sen} \theta} d \theta
$$

Finalmente, las ecuaciones que permiten calcular la matriz de impedancias para una geometría cónica son (Neri, 1999):

$$
Z_{m}=\left.\left.\frac{j \omega \mu}{4 \pi} \Delta\right|_{n} \Delta\right|_{m} \psi(n, \hat{m})+\frac{1}{4 \pi \varepsilon j \omega}
$$

$\left[\Psi\left(\mathrm{n}^{+}, \hat{\mathrm{m}}^{+}\right)-\Psi\left(\mathrm{n}^{-}, \hat{\mathrm{m}}^{+}\right)-\Psi\left(\mathrm{n}^{+}, \hat{\mathrm{m}}^{-}\right)+\Psi\left(\mathrm{n}^{-}, \hat{\mathrm{m}}^{-}\right)\right]$

donde las integrales $\psi$ son del tipo:

$\Psi(n, \hat{m})=\frac{p_{\lambda}}{\Delta l_{n}} \int_{\Delta_{n}} \cos \beta \frac{\exp (-j 2 \pi E)}{F} \sqrt{1-e^{2} \operatorname{sen}^{2} \theta_{n}} d \theta_{n}$

$$
E=
$$

$\frac{\sqrt{r_{m}^{2}\left(1-e \cos \theta_{n}\right)+p_{\lambda}^{2}-2 r_{m} p_{\lambda}\left(1-e \cos \theta_{n}\right) \cos \left(\theta_{m}-\theta_{n}\right)}}{\left(1-e \cos \theta_{n}\right)}$

y

$$
\mathrm{F}=
$$

$\sqrt{r_{m}^{2}\left(1-e \cos \theta_{n}\right)+p_{\lambda}^{2}-2 r_{m} p_{\lambda}\left(1-e \cos \theta_{n}\right) \cos \left(\theta_{m}-\theta_{n}\right)}$
Ahora bien, como se desea realizar un análisis comparativo entre varias geometrías curvas para presentar los resultados con gráficas de fácil comprensión, conviene usar como vari able al radio de curvatura $\rho$, que es el inverso de la curvatura k (Granville, 1995), es decir:

$$
\rho(\theta)=\frac{1}{k(\theta)}
$$

en donde k, para coordenadas polares, es igual a:

$$
k(\theta)=\frac{r^{2}+2 r^{\prime 2}-r r^{\prime \prime}}{\left(r^{2}+r^{\prime 2}\right)^{2}}
$$

donde $r^{\prime}$ y $r^{\prime \prime}$ son respectivamente, la primera y segunda derivada de la magnitud del radio vector $\mathbf{R}\left(\theta_{n}\right)$, cuya posición varía conforme se desplaza el punto fuente desde $n$ - hasta $n+$ a lo largo del segmento $\mathrm{n}$.

Por lo tanto, al derivar la ecuación (1) dos veces y sustituir en la ecuación (14), se tiene que:

$$
\rho(\theta)=\frac{p_{\lambda}\left(1-2 e \cos \theta+e^{2}\right)^{3}}{(1-e \cos \theta)^{3}}
$$

y los resultados mostrados más adelante están en términos de esta variable.

\section{Ejemplo para una geometría parabólica}

Una de las geometrías curvas más empleadas para el diseño de antenas es la parabólica, ya sea con superficie lisa y continua, o bien, integrada por varios tubos parabólicos (Neri y Maclean, 1978). Una forma de analizar las propiedades del enrejado de una antena parabólica es por medio del Método de Momentos, donde hay que cal- 
cular, como primer paso, la matriz de impedancias. Considerando la geometría de la figura 4, la ecuación que representa a la curva parabólica es:

$$
y^{2}=4 a x
$$

o bien:

$$
x=\frac{y^{2}}{4 a}
$$

en donde a es la distancia del vértice al foco de la parábola.

Para conocer todos los términos que se requieren en el cálculo de la matriz de impedancias $\left(\boldsymbol{R}, \mathbf{R}_{m}, \cos \beta\right.$ y dl) se puede sustituir $\mathrm{e}=1$ (excentricidad de la parábola) en las ecuaciones (3), (5), (7) y (12). Estas expresiones

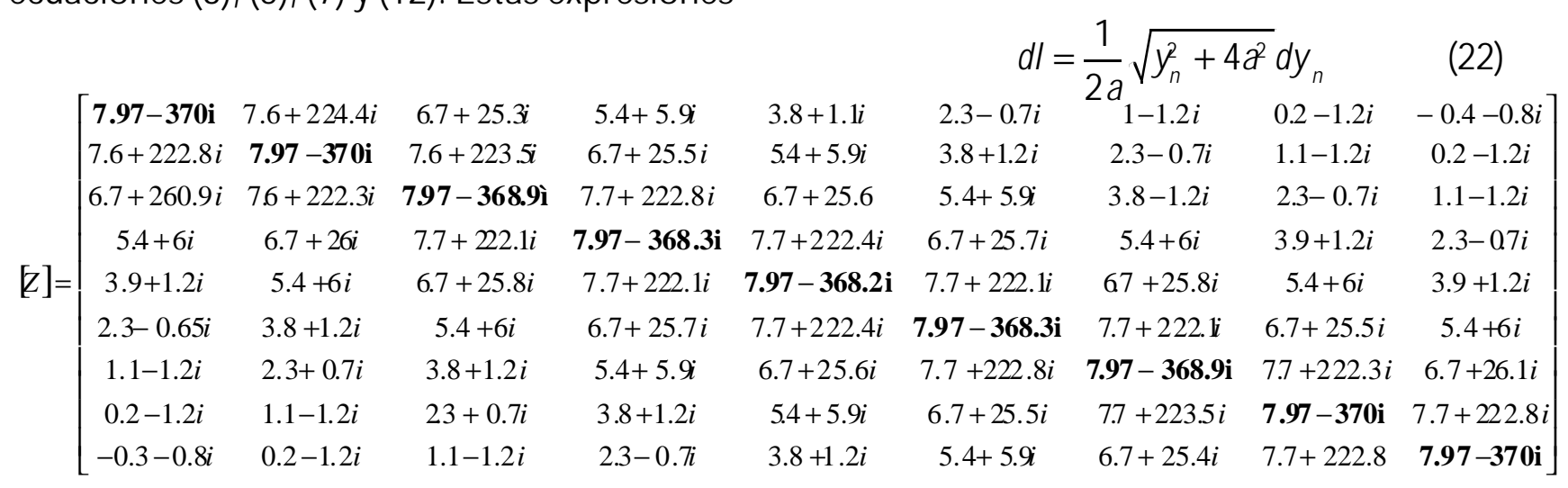

ya simplificadas y escritas en coordenadas cartesianas son:

$$
\mathbf{R}(y)=\frac{y^{2}}{4 a} \mathbf{a}_{x}+y \mathbf{a}_{y}
$$

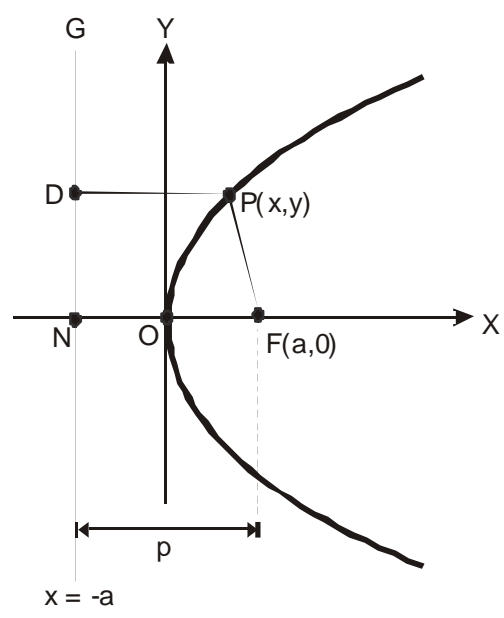

Figura 4. G eometría de un conductor parabólico 
Al programar estas expresiones, es posible obtener la matriz de impedancias para una antena tu bu lar con geometría parabólica por el método de momentos (Neri, 1999). A continuación se muestra esta matriz, cuando $a=1 \lambda$ (distancia focal), la longitud total axial de la antena es $0.6 \lambda$, el diámetro del tubo es $0.01 \lambda, f=300 \mathrm{MHz}$ y $\Delta \mathrm{I}_{n}=0.1 \lambda$.

Las impedancias propias han sido escritas con negritas para resaltarlas. Nótese que los mismos valores de éstas pueden ser obtenidos de las gráficas de la figura 7 en combinación con la figura 5, conociendo la excentricidad y el radio de curvatura del segmento en estudio. El radio de curvatura para un segmento parabólico se obtiene al sustituir e $=1$ y $p=2 a$ en la ecuación (16), o bien, conociendo la posición de dicho segmento, por medio de la fórmula (Granville, 1995):

$$
\rho(y)=\frac{\left(4 a^{2}+y^{2}\right)^{\frac{3}{2}}}{-4 a^{2}}
$$

en la cual, el signo negativo únicamente in dica que la posición del foco se encuentra a la derecha del vértice, como en la figura 4. El lector interesado en conocer el procedimiento matemático para obtener los cam pos radiados por uno 0 varios tubos parabólicos en conjunto puede consultar (Neri, 1978).

\section{Simulación y resultados}

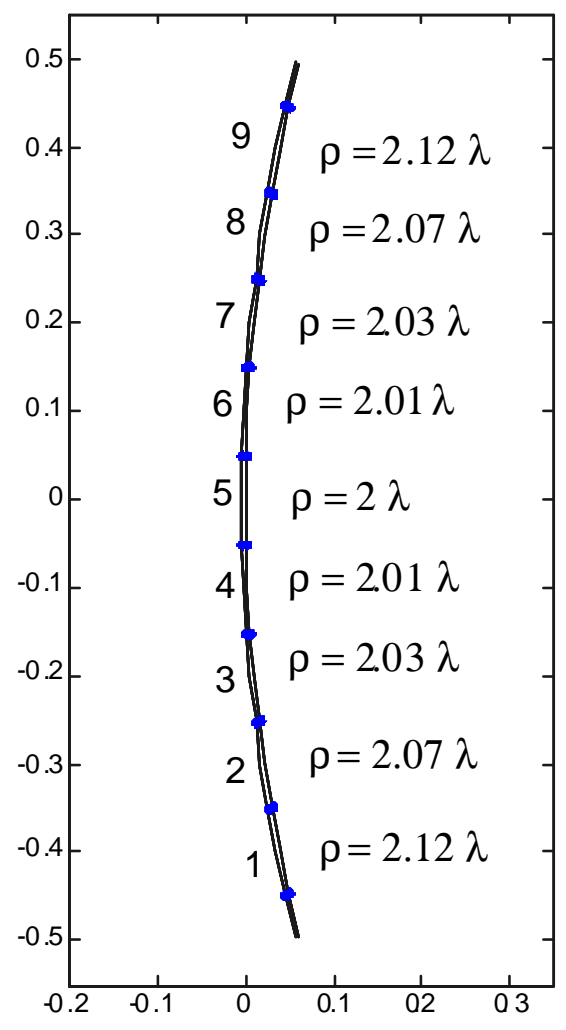

Figura 5. División de un conductor parabólico de longitud $=1 \lambda$ en nueve segmentos de $0.1 \lambda$ y dos semisegmentos extremos de $0.05 \lambda$. Cada segmento está designado con su número correspondiente y a su derecha se indica su radio de curvatura promedio en longitudes de onda 
En la figura 6 se muestran varios dipolos cortos o filamentos de longitud $\Delta=0.1 \lambda$, con diferentes radios de curvatura $(\rho)$, trazados sobre un plano con coordenadas $x$, y en lon gitudes de onda. Al programar las ecuaciones descritas en este trabajo para diferentes ra dios de curvatura, se obtuvieron las gráficas mostradas en la figura 7.

Se observa que el valor de la impedancia propia de cada segmento está en función de la excentricidad de la cónica a la que pertenece y a su radio de curvatura, según su posición sobre la cónica. La excentricidad es una característica de la curva, mientras que el radio de curvatura es una propiedad puntual, por lo que en una misma cónica se pueden tener segmentos cortos con diferentes radios de curvatura.

Para este análisis se consideraron tres valores diferentes de excentricidad: los dos casos extremos y un caso promedio. Los casos extremos son $e=0$ (que representa una circunferencia) y $e=1$ (que describe a una

$\lambda$

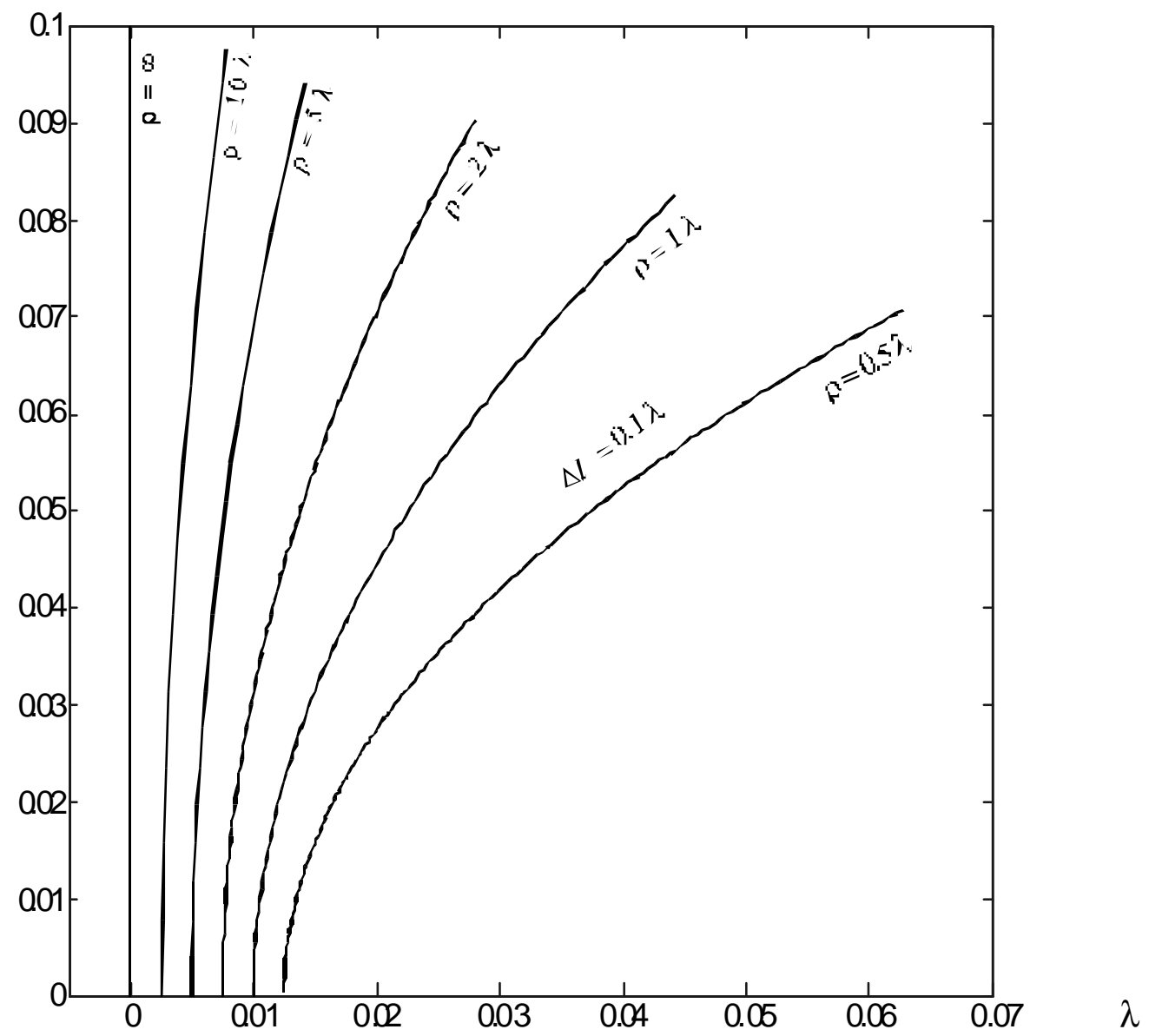

Figura 6. Dipolos cortos 0 segmentos de longitud $\Delta l=0.1 \lambda$ y con diferentes radios de curvatura $\rho$, que definen al eje (segmento $\mathrm{n}) \mathrm{o}$ a la superficie (segmento $\mathrm{m}$ ) del modelo de la figura 3 

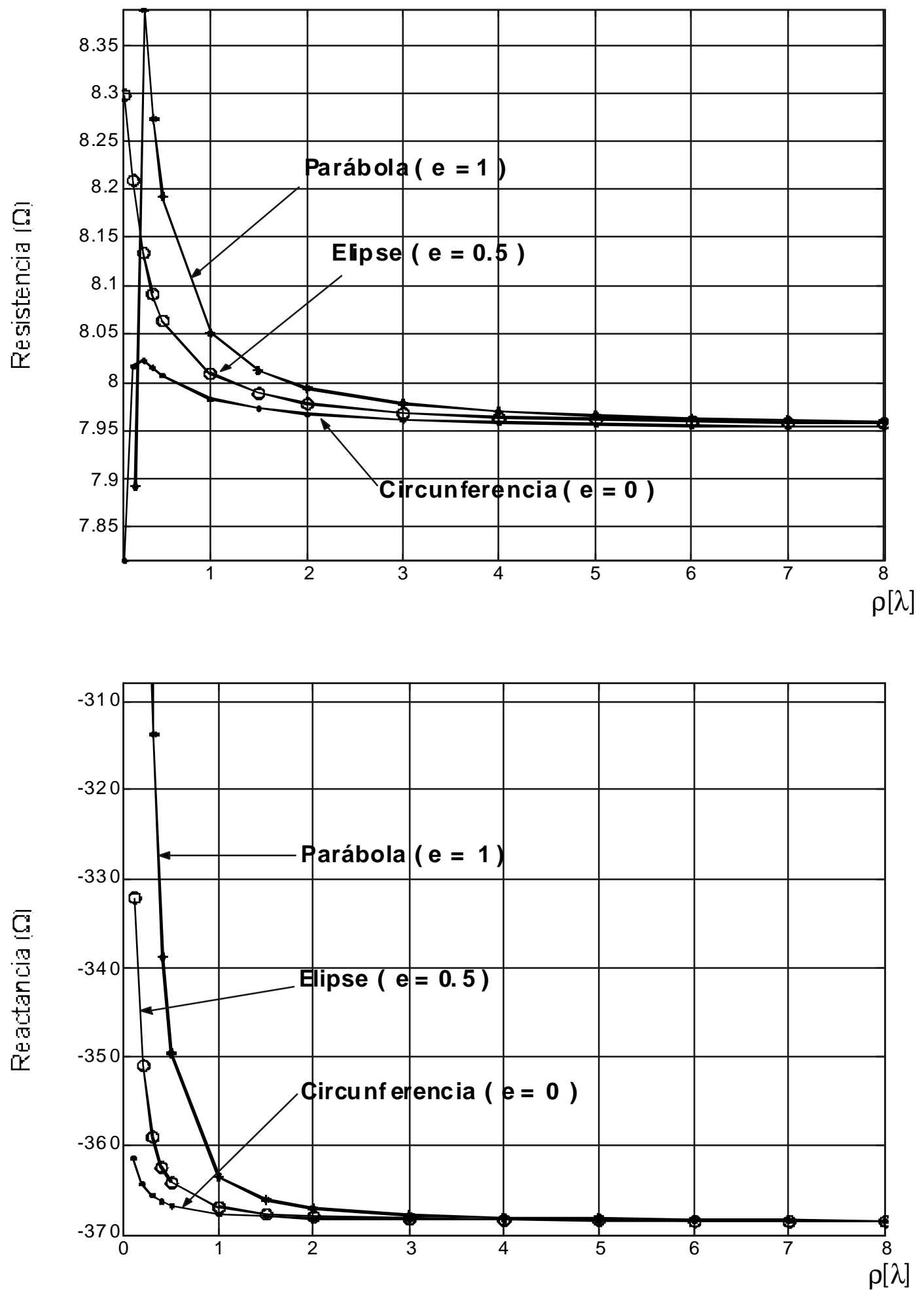

Figura 7. Impedancia propia de un dipolo corto con geometría cónica en función de su radio de curvatura $\rho$ (ecs. 10 y 12) y excentricidad e. Diámetro del conductor $=0.01 \lambda$ y longitud $\Delta=0.1 \lambda$ 
parábola), y el caso promedio es e $=0.5$ (cuyo valor corresponde a una elipse). El radio de curvatura se evaluó en el punto central de cada segmento. Para el caso especial de la circunferencia, todos los puntos sobre ella tienen el mismo radio de curvatura; para variar $\rho$ es necesario modificar el radio de la circunferencia, ya que de acuerdo con la ecuación 16, sustituyendo $e=0$, resulta que $\rho=p_{\lambda}=$ radio.

De la figura 7 se observa que, cuando el radio de curvatura es aproximadamente $0.5 \lambda$, se tiene un valor máximo de la resistencia propia del dipolo, mientras que para radios de curvatura grandes tiende a $7.95[\Omega]$, que es la misma resistencia de un segmento recto, con la misma longitud y diámetro (Neri, 1980). Se puede concluir entonces que la resistencia aumenta al disminuir el radio de curvatura, pero cuando este radio es menor que aproximadamente 0.5 lambda y tiende hacia cero, hay una caída brusca de la resistencia.

Esto se debe a que cuando los dipolos están fuertemente doblados (con pequeño radio de curvatura), las diferentes partes del dipolo producen los campos contra-restantes en la zona lejana de radiación. En cuanto a la parte imaginaria de la impedancia propia, también se observa que, conforme $\rho$ aumenta, la reactancia (que es capacitiva) converge a un valor constante de $-367[\Omega]$ e igual al de un dipolo recto (Neri, 1980). Se concluye que si el radio de curvatura es igual o mayor que aproximadamente 2 , por lo que se refiere a las impedancias propias, todo segmento curvo de una antena con geometría cónica puede ser considerado como recto sin que se introduzca ningún error significativo en la matriz de impedancias. Pero conforme el radio de curvatura se reduce hacia 10 menos, es imprescindible modelar los segmentos con su curvatura correcta, ya que aproximarlos por dipolos rectos introduciría errores del orden de $5 \%$ y $18 \%$ en la resistencia y la reactancia, respectivamente. Estos errores tendrían un efecto acumulativo aún mayor al invertir la matriz, por lo que las corrientes obtenidas no serían las correctas, dado que éstas quedan determinadas por una de las columnas de la matriz inversa. Otra solución cuando el radio de curvatura es muy pequeño sería usar segmentos 0 dipolos con longitudes menores que $0.1 \lambda$, siempre y cuando se respete la regla de que el cociente longitud/diámetro de cada segmento sea del orden de 50 mayor (Neri y Mclean, 1978 bis).

Asimismo, se observa que la cónica que tiene una mayor variación en las impedancias propias de sus elementos es la parábola $(e=$ 1 ), ya que presenta valores muy altos para una $\rho$ pequeña y converge hacia $7.95 \quad[\Omega]$ aproximadamente para $\rho>4$. Por el contrario, la curva que posee el comportamiento más estable para un mayor rango de $\rho$, es la circunferencia.

\section{Conclusiones}

La resistencia propia de un dipolo curvo decae exponencialmente en función de su radio de curvatura, mientras que la reactancia propia (de tipo capacitivo) crece exponencialmente; ambas componentes de la impedancia propia convergen muy rápido para $\rho \geq 2$ hacia la impedancia propia de un dipolo recto. Por lo tanto, cualquier antena con geometría cónica puede ser modelada por el método de momentos, segmentándola con dipolos rectos, siempre y cuando $\rho \geq 2$. Esta información puede ser muy útil para alumnos, profesores e investigadores que escriban sus propios programas de computadora para analizar antenas con geometría cónica.

\section{Referencias}


Antón H. (1998). Introducción al álgebra lineal. Edito rial Limusa, México.

Granville W.A. (1995). Cálculo diferencial e integral. Noriega Editores, México.

Harrington R.F. (1968). Field Compu ta tion by M oment M ethods. Macmillan, Nueva York. Hernández V., Valiente L.A. y Neri R. (2001). Input Impedance, Current Distributions and Radiation Properties of Thick and Widely Spaced Folded Dipoles. Electromagnetics, Vol. 21, No. 5, pp. 435 - 449.

Neri R. y Maclean T.S.M. (1978). On-axis

Fields of Small Grid Paraboloid by Moment Method. Microwave, 0 ptics and A coustics. IEE, Vol. 2, No. 4, Inglaterra. Neri R. y Maclean T.S.M. (1978 bis). Axial-Line-Current Approximation for
Short Cylindrical Wires. Electronics Letters, Vol. 14, No. 24, pp. 776 - 778.

Neri R. (1980). Self and Mutual Impedances Between Sub-Elements of Dipole Antennas: Contributions from Currents and Charges. Int. J. Electronics, Vol. 48, No. 5, pp. $435-442$.

Neri R. (1999). Análisis de antenas lineales por el Método de Momentos. Ingeniería Investigación y Tecnología, Vol. I, No.2, enero- marzo, UNAM, pp. 73 -

0'§̊̉̉il P.V. (1999). Matemáticas avanzadas para ingeniería. 3ạ. edición, Vol. 2, CECSA, México.

Solis R., Nolasco J. y Victoria A. (1995). Geometría analítica. Limusa, Noriega Editores, 5a edición, México.

Spiegel M.R., Liu J. y Avellanas L. (2000). Fórmulas y tablas de matemática aplicada. McGraw-Hill, 2ª edición, España.

\section{Semblanza de los autores}

A lejandro M onsiváis-H uertero. Entre los años de 1997 y 2002 cursó en la Facultad de Ingeniería de la UNAM la carrera de ingeniería en telecomunicaciones con la especialidad de dispositivos de microondas. Ha tomado diferentes cursos en la Fundación TELMEX y actualmente realiza sus estudios de doctorado en Francia becado por el Conacyt.

Rodolfo N eri-Vela. Realizó los estudios de ingeniero mecánico electricista y se especializó en telecomunicaciones y electrónica en la Facultad de Ingeniería, UNAM. En 1976, finalizó la maestría en telecomunicaciones en la Universidad de Essex, Inglaterra, becado por el Consejo Británico. Tres años después obtuvo el grad o de doctor en radiación electromagnética aplicada por la Universidad de Birmingham, Inglaterra, como becario del Consejo Nacional de Ciencia y Tecnología (CONACYT). En 1985, se convirtió en el primer astronauta mexicano al participar en la misión 61-B de la NASA de los EU y orbitar la Tierra 109 veces. Actualmente imp arte cátedra en el Departamento de Telecomunicaciones de la Facultad de Ingeniería de la UNAM. 